\title{
Effect of Pulsed Magnetic Field on the Residual Stress of Rolled Magnium Alloy AZ31 Sheet
}

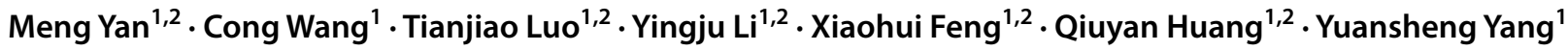

Received: 26 March 2020 / Revised: 11 May 2020 / Accepted: 25 May 2020 / Published online: 25 July 2020

(c) The Chinese Society for Metals (CSM) and Springer-Verlag GmbH Germany, part of Springer Nature 2020

\begin{abstract}
A novel method of pulsed magnetic field (PMF) treatment was developed to eliminate the residual stress of rolled magnesium alloy AZ31 sheet in this study. The effect of PMF on residual stress of rolled AZ31 sheet was investigated and its mechanism was analyzed. The experimental results revealed that the pulse frequency had a significant impact on residual stress. After $10.0 \mathrm{~Hz}$ PMF treatment, the average and maximum reduction rates of residual stress along the rolled direction were $26.6 \%$ and $30.3 \%$, respectively. It was found that the dislocation density and parallel dislocation in grains of the rolled sheet increased after it was treated by the pulsed magnetic field. The simulation results showed that the Lorentz force generated by the pulsed magnetic field can lead to basal slip, thereby resulting in local plastic deformation. Besides, the Joule heat produced during the PMF treatment was conducive to the elimination of residual stress.
\end{abstract}

Keywords Wrought magnesium alloy $\cdot$ Residual stress $\cdot$ Pulsed magnetic field $\cdot$ Rolled AZ31 sheet

\section{Introduction}

In recent years, transportation sectors have been making more and more efforts to reduce vehicle weight because energy conservation and emission reduction are economically and environmentally beneficial $[1,2]$. In such context, magnesium alloys have attracted extensive attention due to their unique low mass density [3]. After extrusion or rolling, magnesium alloys will exhibit better mechanical properties due to their refined microstructure and alleviated cast defects $[4,5]$. However, limited slip systems and inhomogeneous plastic strain will inevitably cause great residual stress in magnesium alloys, thus further leading to problems including distortion failure, stress-corrosion cracking, and low fatigue life, as well as poor production quality and high costs [6-10]. Therefore, it is of great importance to remove such residual stress. So far, a number of methods

Available online at http://link.springer.com/journal/40195.

Yuansheng Yang

ysyang@imr.ac.cn

1 Institute of Metal Research, Chinese Academy of Sciences, Shenyang 110016, China

2 School of Materials Science and Engineering, University of Science and Technology of China, Hefei 230026, China such as natural aging, annealing [11], vibratory stress relief [12-14], and uphill quenching [15, 16] have been employed to eliminate residual stress in steel and aluminum alloys. Among them, annealing has also been used to eliminate the residual stress in magnesium alloys $[17,18]$. In view of the detriment of residual stress and the promising application prospect of magnesium alloys, it is necessary to develop more efficient methods of removing residual stress in magnesium alloys. Amid the above methods, heat or mechanical force has been used for reducing elastic strain and generating plastic deformation effectively. Therefore, the combination of heat and mechanical force can be reasonably assumed to be an more efficient solution. As is known to all, according to the Maxwell equations, Lorentz force and Joule heat can be generated simultaneously when an electromagnetic field acts on metals [19]. Thus, the effect of electromagnetic method on residual stress has attracted much attention in recent years. A few of studies investigated the effects of pulsed magnetic field (PMF) on stress state and microstructure of alloys. Lu et al. [20] found that strong pulsed magnetic treatment resulted in stress relaxation in both rigid restrained tensile specimens and welded specimens of HT50 steel. In addition, magnetic treatment cycles affected the stress reduction ratio. Klamecki et al. [21] reported that $4 \%-7 \%$ of stress was reduced on the surface of low-stress steel specimens, while $8 \%-13 \%$ of stress was reduced on the 
surface of high-stress ones. Wu et al. [22] considered that the stress relaxation of $30 \mathrm{CrMnSiA}$ medium carbon steel was mainly caused by the magnetostriction effect. Cai et al. [23] explained that the residual stress of steel samples was eliminated mainly because the dislocation depinning was facilitated by the magnetic field and the ability of conduction electrons to drive the dislocation further and faster was improved by pulsed current. Song et al. [24] proposed that the magneto-plastic deformation and the change of magnetic domains were the fundamental causes of the stress relaxation in low alloy steel. Tang et al. [25] considered that PMF changed the state of radical pairs to increase T-state radical pairs, which made dislocation mobility enhanced. It is worth noting that the above research works primarily focus on the reduction of residual stress of steels. The explanation of the stress relaxation is qualitative and lack of consistent. Therefore, plenty of issues need to be further addressed so as to have a better understanding of the effect of pulsed magnetic field on residual stress. Considering the joint role of heat and force produced by the pulsed magnetic field, we investigated the effect of PMF parameters on the residual stress of rolled magnesium alloy sheet. The present work mainly studied the residual stress and microstructure evolution of rolled mganesium alloy AZ31 sheet under PMF and explained the mechanism of stress change in magnesium alloy.

\section{Experimental}

A rolled AZ31 sheet with the elemental composition (wt.\%) of $\mathrm{Al} 3.37 \%, \mathrm{Zn} 0.86 \%$, Mn $0.29 \%$, and $\mathrm{Mg}$ bal. was used as raw material for the experiment. The sheet was rolled from 12 to $4 \mathrm{~mm}$ thick at $450{ }^{\circ} \mathrm{C}$, and the samples used for stress measurement were taken from this sheet using the electrical discharge machining method. The size of the sample and the positions $(-15,0),(0,0)$ and $(15,0)$ for stress measurement are shown in Fig. 1, in which RD, TD, and ND represent

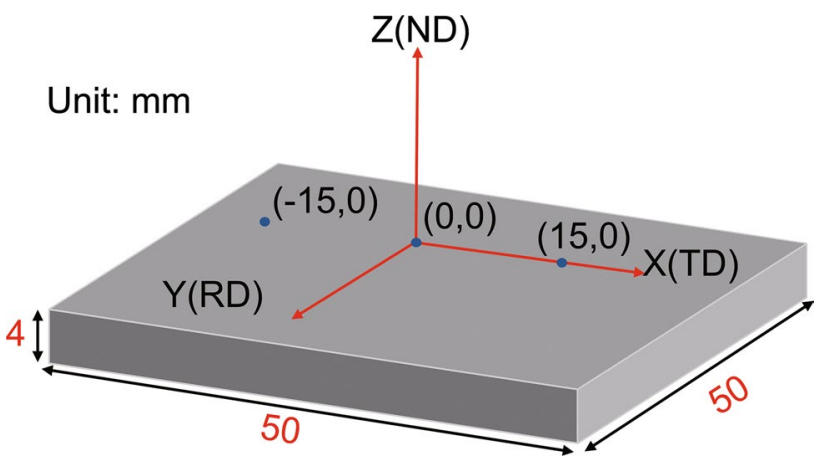

Fig. 1 Dimension of the specimen and the positions for stress measurement rolling direction, transverse direction, and normal direction, respectively.

The apparatus of PMF treatment is illustrated in Fig. 2. The apparatus is composed of a low-voltage pulsed power source, exciting coils, a stainless steel holder, and a cooling water system. The pulsed magnetic field generated by the exciting coils acted on the magnesium alloy AZ31 sheet sample. A cooling system was used to cool the coils and isolate the heat generated in the coils from the sample.

The PMF voltage was fixed at $300 \mathrm{~V}$ and the treatment time was 60 minutes in the experiment. Besides, the PMF frequency was set at $2.5,5.0$, and $10.0 \mathrm{~Hz}$ to investigate the effect of the frequency on residual stress. The sample was placed in the center of the exciting coils for PMF treatment after the initial residual stress was tested.

The temperature rise on the sample surface was measured by a thermocouple during the treatment to evaluate the effect of Joule heat on residual stress. In order to assess the effect of Lorentz force on residual stress, a forced cooling system was used to cool down the sample to room temperature.

The Lorentz force was simulated by the finite element software ANSYS. And a 3D model was built and the solid 97 elements were selected. The resistivity of the magnesium and copper coils was $4.46 \times 10^{-8} \Omega \mathrm{m}^{-1}$ and $2 \times 10^{-8} \Omega \mathrm{m}^{-1}$, respectively, and the relative magnetic permeability of air, coils, and sample was set at 1 . The power with a voltage of $300 \mathrm{~V}$ and a frequency of $10.0 \mathrm{~Hz}$ was applied to the coils, as shown in Fig. 3. Each cycle was composed of a rising duration of $5 \mathrm{~ms}$, a falling duration of $50 \mathrm{~ms}$, and a pulse interval of $45 \mathrm{~ms}$.

After PMF treatment, the residual stress along RD and TD at positions $(-15,0),(0,0)$, and $(15,0)$ was measured again by X-ray diffraction (XRD) with Co anode and a beam diameter of $1.5 \mathrm{~mm}$. Then the residual stress was calculated by the $\sin ^{2} \psi$ method [26] under the assumption of biaxial stress state according to Eq. (1):

$$
\begin{aligned}
\frac{d_{\varphi \psi}-d_{0}}{d_{0}}= & \frac{1+v}{E}\left(\sigma_{11} \cos ^{2} \varphi+\sigma_{12} \sin 2 \varphi+\sigma_{22} \sin ^{2} \varphi\right) \\
& \sin ^{2} \psi-\frac{v}{E}\left(\sigma_{11}+\sigma_{22}\right) .
\end{aligned}
$$

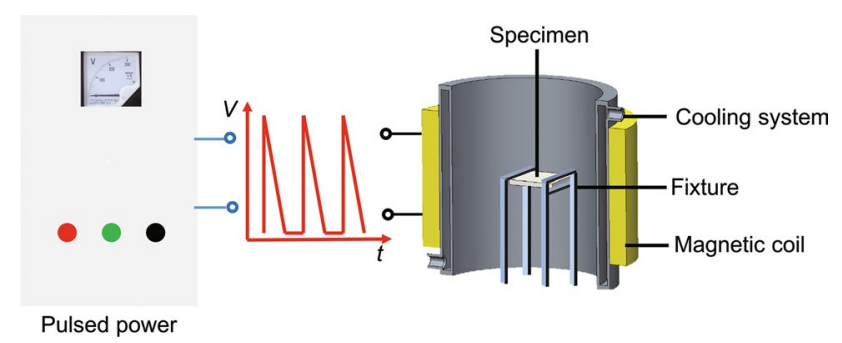

Fig. 2 Schematic illustration of PMF apparatus 


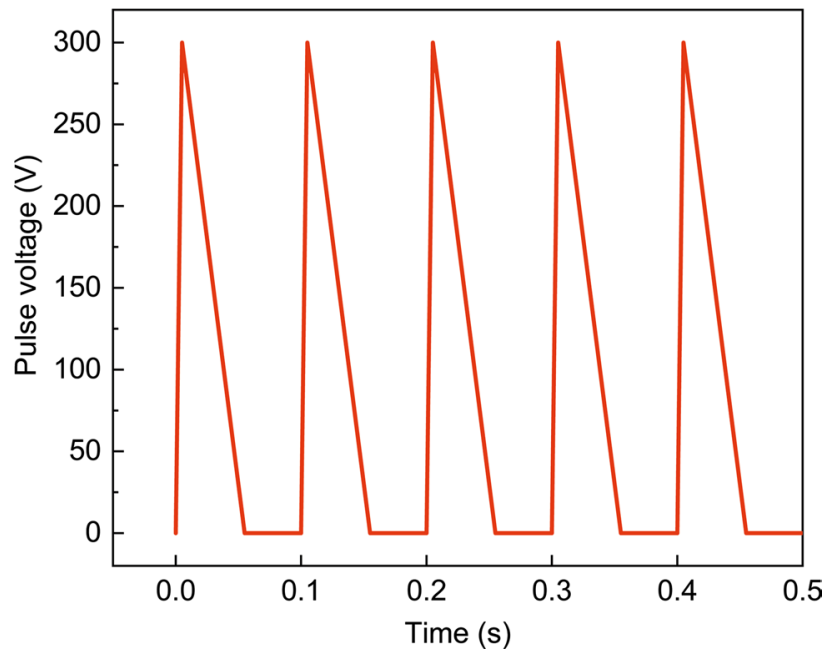

Fig. 3 Pulse voltage waveform of $10.0 \mathrm{~Hz}$ PMF

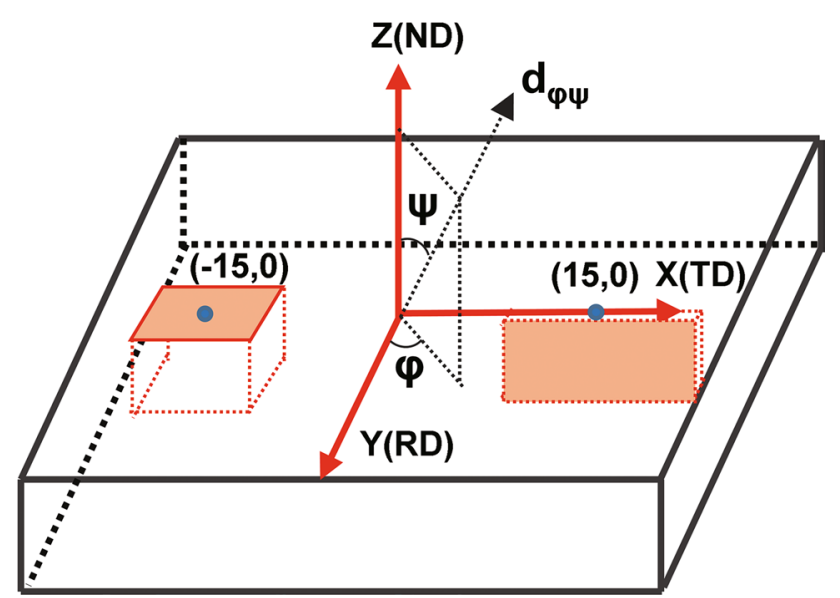

Fig. 4 Schematic showing the measurement of residual stress by XRD and sampling location for EBSD and TEM

where $d_{\varphi \psi}$ is the measured spacing of diffracting plane with its normal determined by $\varphi$ and $\psi$, as shown in Fig. $4, d_{0}$ is the lattice spacing in an unstressed sample, $v$ is Poisson's ratio, and $E$ is the macroscopic elastic modulus. The plane spacing of $(20 \overline{2} 4)$ at different $\varphi\left(0^{\circ}\right.$ and $\left.90^{\circ}\right)$ and $\psi\left(0^{\circ}, \pm 9.2\right.$ $\left.9^{\circ}, \pm 19.5^{\circ}, \pm 29.71^{\circ}, \pm 39.00^{\circ}\right)$ was recorded. Subsequently, the residual stress along RD and TD was calculated from the slope of $d_{\varphi \psi-} \sin ^{2} \psi$ according to Eq. (1). Each point was tested for three times, and the average value was calculated as the final value to reduce accidental errors.

The samples for microstructure characterization were cut from the specimen treated by PMF. As shown in Fig. 4 , with $(-15,0)$ as the center, a square sample with a dimension of $5 \mathrm{~mm} \times 5 \mathrm{~mm} \times 4 \mathrm{~mm}(\mathrm{RD} \times \mathrm{TD} \times \mathrm{ND})$ was cut off for electron backscattered diffraction (EBSD) observations, and with $(15,0)$ as the center, a rectangular sample with a dimension of $0.1 \mathrm{~mm} \times 10 \mathrm{~mm} \times 4 \mathrm{~mm}$ $(\mathrm{RD} \times \mathrm{TD} \times \mathrm{ND})$ was cut off for transmission electron microscopy (TEM) characterization.

The samples for EBSD observations were ground using $\mathrm{SiC}$ paper then polished with a $2.5 \mu \mathrm{m}$ diamond paste and then with an electrolytic polishing solution containing 10 vol.\% perchloric acid and 90 vol.\% alcohol. Kernel average misorientation (KAM) map was characterized by EBSD on a Zeiss Merlin Compact microscope with 1000 magnification and $0.5 \mu \mathrm{m}$ step size. The TEM samples were prepared using an ion thinner with a cold stage to reduce the influence of temperature rise on dislocation. The dislocation configuration before and after PMF treatment was characterized by a Tecnai G20 transmission electron microscope.

\section{Results and Discussion}

\subsection{Effect of PMF Treatment on Residual Stress}

The residual stress along RD and TD of the AZ31 sheet before and after PMF treatment is shown in Fig. 5a-c. As can be seen from the figure, the residual stress along both RD and TD is compressive and nonuniformly distributed. After PMF treatment, the stress along RD and TD was apparently released and uniformly distributed with the increase of PMF frequency. The residual stress at three positions and along $\mathrm{RD}$ and TD was eliminated by PMF treatment to varying degrees. Specifically, more stress was eliminated at the positions $(15,0)$ and $(-15,0)$ than at the position $(0,0)$, and the residual stress along rolling direction was more effectively eliminated than that along the transverse direction. Besides, PMF frequency is also an influencing factor in eliminating residual stress. As PMF frequency increases, the value of elimination of residual stress goes up, as shown in Fig. 5d. The residual stress was the most notably eliminated after 10.0 Hz PMF treatment, with an average and maximum reduction rate of $26.6 \%$ and $30.3 \%$, respectively.

\subsection{Effect of PMF Treatment on Microstructure}

\subsubsection{Strain Distribution}

The KAM maps of the samples without and with being treated by $10.0 \mathrm{~Hz}$ PMF are shown in Fig. 6. Comparing Fig. $6 a$ and b, there are obvious differences in the KAM values and distributions between these two samples. For the untreated sample, high-value KAM areas are very small and concentrate in a few grains. However, the high-value KAM of the sample with being treated by PMF are uniformly distributed in a large areas. Specifically, the average KAM 

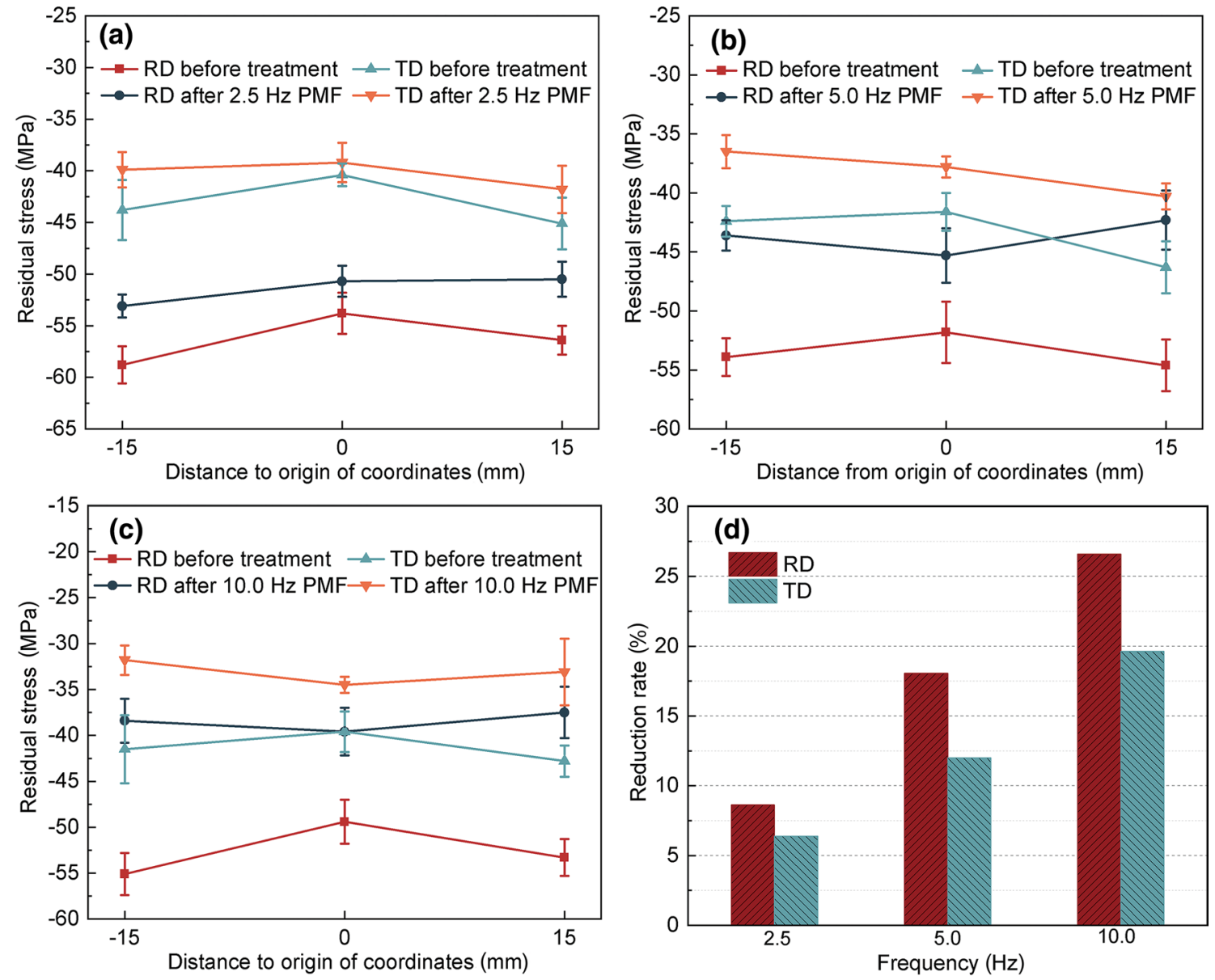

Fig. 5 Effects of PMF frequency on RD and TD residual stress: a $2.5 \mathrm{~Hz}, \mathbf{b} 5.0 \mathrm{~Hz}, \mathbf{c} 10.0 \mathrm{~Hz}$ and d average and maximum reduction rate of residual stress
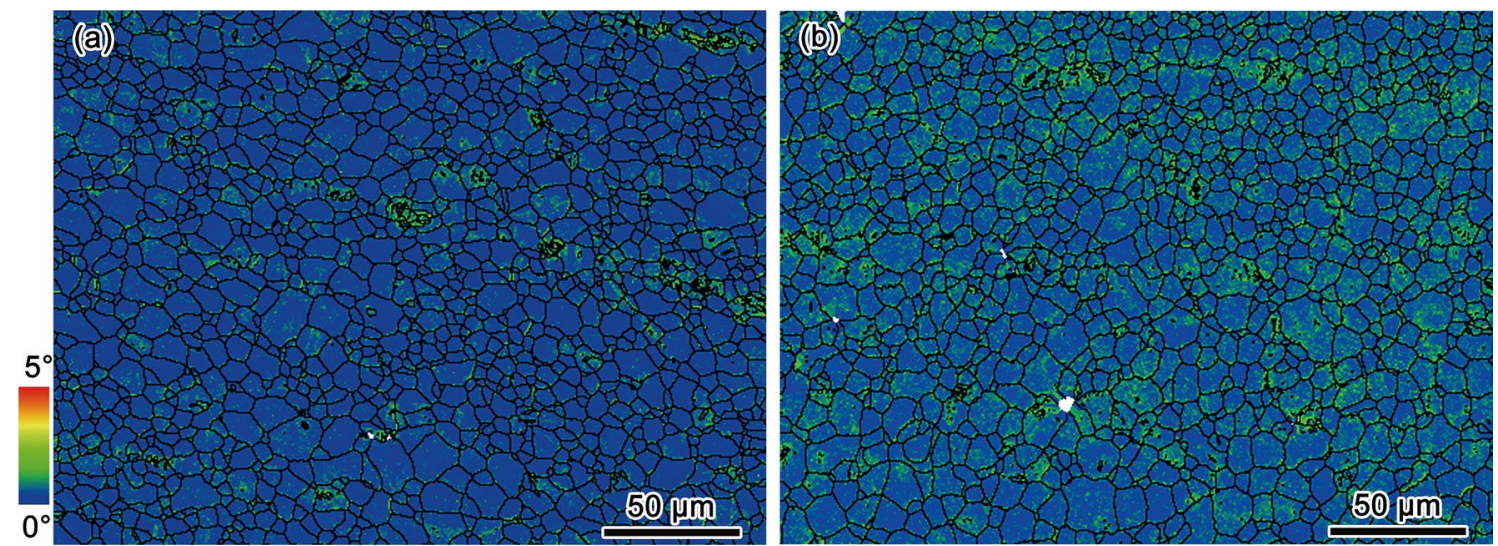

Fig. 6 KAM maps of $\mathbf{a}$ untreated and $\mathbf{b}$ with $10.0 \mathrm{~Hz}$ PMF treatment specimens

value is increased from $0.5^{\circ}$ to $0.65^{\circ}$ after $10.0 \mathrm{~Hz} \mathrm{PMF}$ treatment.

KAM value represents the extent of plastic deformation. The higher the value, the more serious the plastic deformation [27, 28]. As shown in Fig. 6a, the KAM value is heterogeneous among grains, with serious deformation concentrated in a few grains. Moreover, the strain within grains is also not uniform. Such uneven plastic deformation within and among grains would result in residual stress [7]. As shown in Fig. 6b, the high-value KAM 
is distributed in a larger area, indicating that local deformation occurred during PMF treatment, thereby enabling the strain to be uniformly distributed and coordinating new internal stress among grains. Thus, the residual stress is eliminated [22].

\subsubsection{Dislocation Configuration}

The dislocation configuration of the basal plane before and after PMF treatment was characterized, as shown in
Fig. 7a-f. According to the figures, the dislocation density is significantly increased after PMF treatment. As can be seen from Fig. 7a, few dislocations were observed in the sample without PMF treatment, but they increased slightly in the sample with being treated by $2.5 \mathrm{~Hz}$ PMF, as shown in Fig. 7b. As the pulse frequency increased from 5.0 to $10.0 \mathrm{~Hz}$, the number of dislocations was crucially multiplied. Besides, a lot of parallel dislocations (marked with yellow rectangles) were identified after $10.0 \mathrm{~Hz}$ PMF treatment,
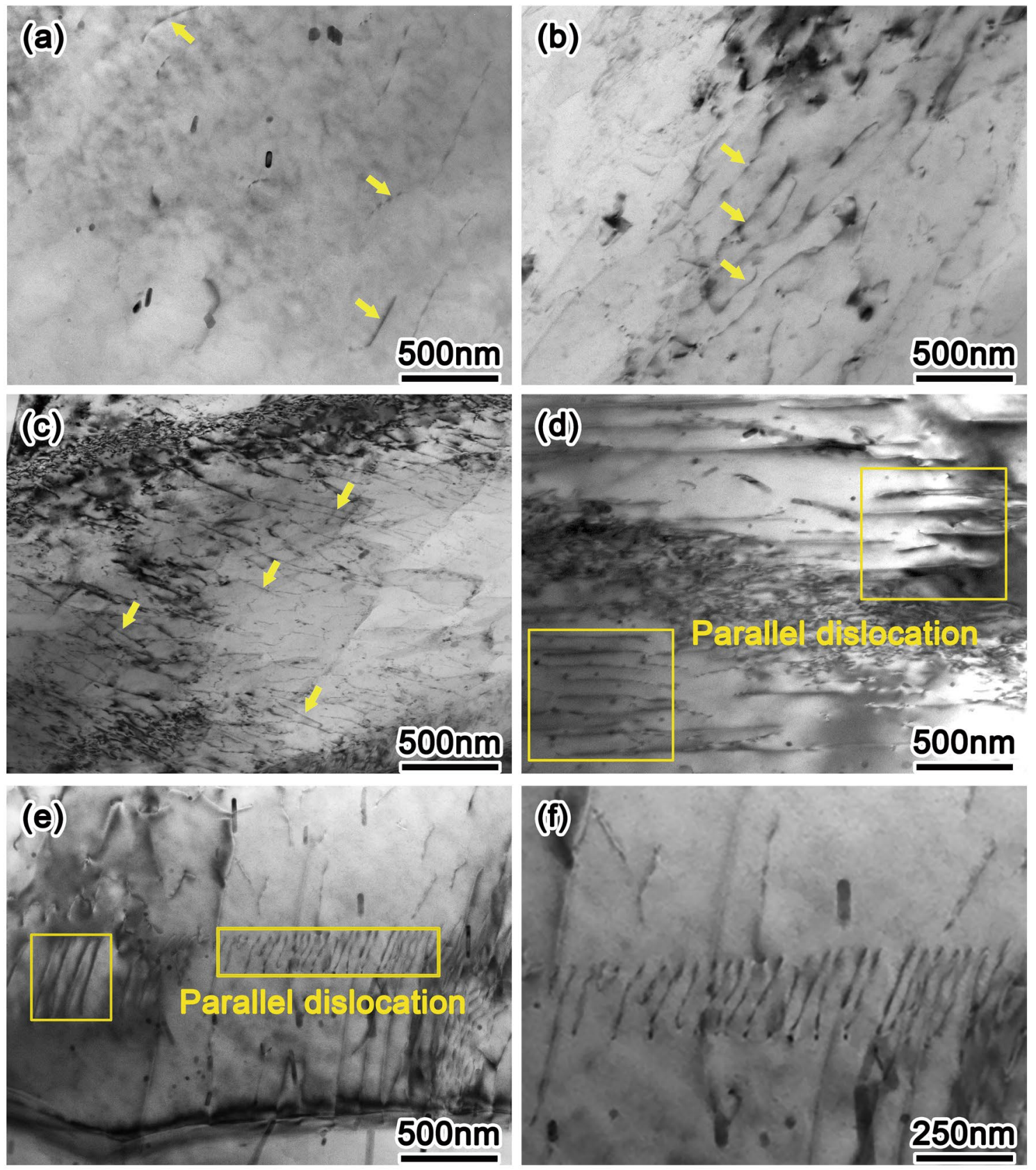

Fig. 7 TEM micrograph of dislocation before a and after b $2.5 \mathrm{~Hz}, \mathbf{c} 5.0 \mathrm{~Hz}, \mathbf{d}, \mathbf{e}, \mathbf{f} 10.0 \mathrm{~Hz}$ PMF treatment 
as displayed in Fig. 7d-f, implying that dislocation moved during PMF treatment [29].

As reported in previous research, the mobile ability of dislocation can be improved by PMF treatment, thus increasing the dislocation density $[30,31]$. The increased dislocation density and altered dislocation arrangement indicate that PMF treatment facilitates dislocation proliferation and movement, thus generating local deformation, which is consistent with the KAM results. The balance of the initial stress will be broken by the dislocation movement in the grains, thus resulting in the redistribution of residual stress.

\subsection{Effect of Lorentz Force on Residual Stress}

The distribution of Lorentz force was simulated by finite element software ANSYS, and the basal slip was analyzed to clarify the mechanism of microstructural evolution under PMF treatment.

As displayed in Fig. 8, the Lorentz force is compressive and parallel to the RD-TD plane at the end of the rising cycle. The Lorentz force near the boundary is maximum, with a value of $1.36 \times 10^{8} \mathrm{~N} \mathrm{~m}^{-3}$, but it gradually decreases from boundary to the center of the sample, which is similar to the simulation results in previous research [32]. By converting volume force to stress with reference to previous research [33], maximum shear stress imposed by the Lorentz force was $6.75 \mathrm{MPa}$. If the resolved shear stress $\tau$ in the slip system is larger than the corresponding critical resolved shear stress $\tau_{\mathrm{c}}$, the slip will be activated by Lorentz force.

The Schmid factor map and the histogram of (0001) $\langle 11 \overline{2} 0\rangle$ slip system are shown in Fig. 9a-d when Lorentz force is parallel to the RD and TD of the sample. The average Schmid factor $m$ of RD and TD was 0.25 and 0.21 , respectively. The resolved shear stress $\tau$ in the slip system

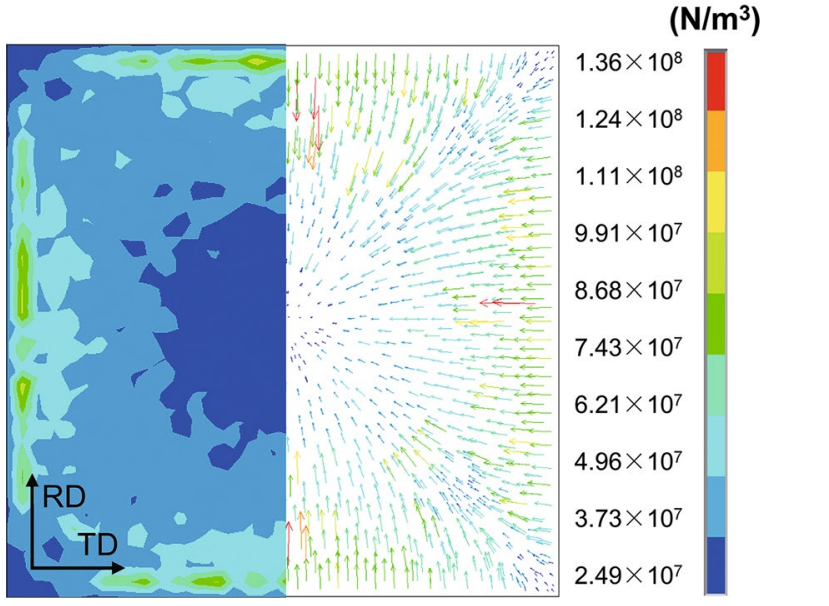

Fig. 8 Lorentz force in the rising period PMF treatment can be calculated according to equation $\tau=\sigma \times m$, where $\tau$ is the resolved shear stress on slip system and $\sigma$ is the stress induced by Lorentz force. Therefore, shear stress along RD and TD was calculated as $1.68 \mathrm{MPa}$ and $1.41 \mathrm{MPa}$, respectively. It was reported that the critical resolved shear stress $\tau_{\mathrm{c}}$ of the single-crystal magnesium basal slip was about 0.5-0.9 MPa [34-36]. It is worth noting that only local plastic deformation occurred, which means that, only grains with large Schmid factor were deformed during PMF treatment. Therefore, it was not macroplastic deformation. For the local plastic deformation in one grain, the $\tau_{\mathrm{c}}$ of single crystal was still effective. By comparing resolved shear stress $\tau$ with $\tau_{\mathrm{c}}$, it can be deduced that the basal slip of the grain with higher Schmid factor can be activated by Lorentz force, thereby generating local plastic deformation and causing dislocation movement and proliferation.

In addition, the resolved shear stress along RD was larger than that of TD, so the basal slip was more likely to occur in RD during PMF treatment. Accordingly, the residual stress was more effectively eliminated along RD. Meanwhile, as shown in Fig. 8, Lorenz force at the positions $(15,0)$ and $(-15,0)$ is larger than that at $(0,0)$, so the basal slip is more likely to start in more grains at the position $(15,0)$ and $(-15,0)$ than $(0,0)$. Therefore, more residual stress was eliminated at these two positions.

The Lorentz force and Joule heat were generated simultaneously during PMF treatment. Therefore, the effect of Joule heat during PMF treatment needs to be excluded so as to quantitatively evaluate the effect of Lorentz force on the residual stress. To prevent the sample from temperature rise during PMF treatment, a forced cooling system was applied. The residual stress and its reduction rate under the action of Lorentz force with different frequencies are listed in Table 1. According to the statistical results, the residual stress in three cases was eliminated, which could be ascribed to basal slip and more uniformly distributed strain initiated by Lorentz force. Meanwhile, as the frequency of Lorentz force increased, the residual stress was eliminated more effectively, which was mainly because more pulses were applied to the sample with a higher frequency, thus more easily promoting local plastic deformation and dislocation movement.

\subsection{Effect of Joule Heat on Residual Stress}

Joule heat generated during PMF treatment can result in temperature rise of the sample, thereby possibly affecting residual stress. The temperature rises on the surface of the sample during PMF treatments at different frequencies are illustrated in Fig. 10a. As described in the figure, the temperature-time curve contains a rising part and a stable part. The temperature rose from $30^{\circ} \mathrm{C}$ to $47.0,64.5$, and $73.8^{\circ} \mathrm{C}$ after $2.5,5.0$, and $10.0 \mathrm{~Hz}$ PMF treatment, respectively. 

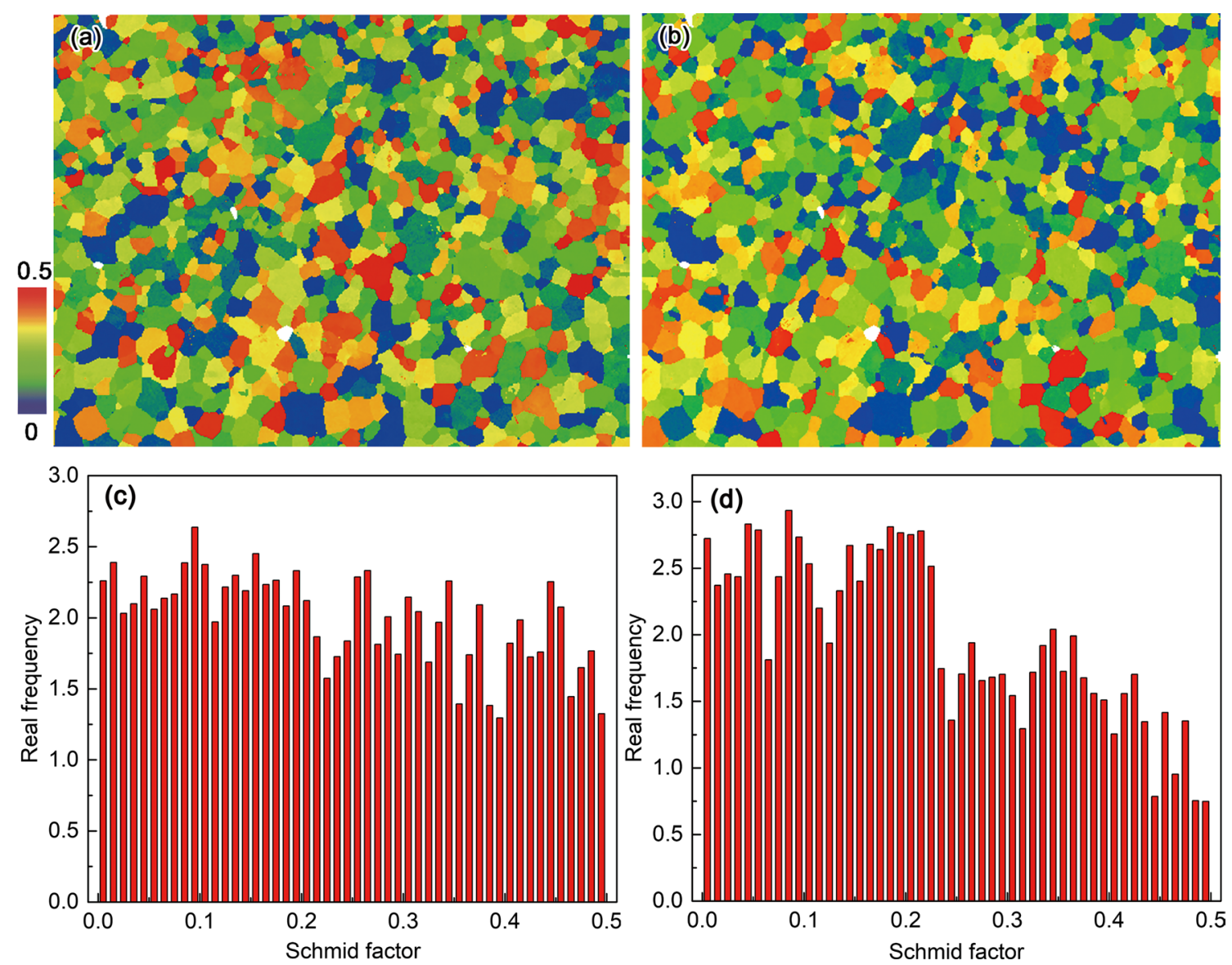

Fig. 9 Schmid factor map and histogram of basal slip a, $\mathbf{c}$ loaded along RD and $\mathbf{b}, \mathbf{d}$ along with TD

Table 1 Residual stress of samples treated with different frequencies Lorentz force

\begin{tabular}{|c|c|c|c|}
\hline \multirow[t]{2}{*}{ Frequency } & \multicolumn{2}{|c|}{ Residual stress (MPa) } & \multirow{2}{*}{$\begin{array}{l}\text { Reduc- } \\
\text { tion rate } \\
(\%)\end{array}$} \\
\hline & Before treatment & After treatment & \\
\hline $2.5 \mathrm{~Hz}$ & $-50.3 \pm 1.7$ & $-47.0 \pm 1.2$ & 6.5 \\
\hline $5.0 \mathrm{~Hz}$ & $-51.2 \pm 2.5$ & $-44.3 \pm 1.4$ & 13.3 \\
\hline $10.0 \mathrm{~Hz}$ & $-52.5 \pm 2.4$ & $-42.9 \pm 1.3$ & 18.1 \\
\hline
\end{tabular}

To assess the effect of Joule heat on the residual stress, the residual stress of another three samples was also measured after they were heated for $1 \mathrm{~h}$ in a muffle furnace at 47.0, 64.5, and $73.8{ }^{\circ} \mathrm{C}$, respectively. The residual stress before and after treatment at the three temperatures is exhibited in Table 2. It can be seen from the table that the residual stress was eliminated slightly after heat treatment. Additionally, its reduction rate gradually increased as temperature rose. The slightly eliminated residual stress may be caused by the enhanced irregular motion of atoms at a higher temperature. Hence, the lattice distortion is likely to be released during heat treatment, and the residual stress is thus eliminated.
The reduction rates induced by Lorentz force and Joule heat were compared, as illustrated in Fig. 10b. As can be seen from the figure, the elimination effect caused by Joule heat is not as remarkable as that by Lorentz force during PMF treatment at different frequencies. Therefore, it is reasonable to infer that Lorentz force is the principal factor for eliminating residual stress.

It was also revealed that the elimination effect was significantly improved as PMF frequency increased mainly for the following reasons. During PMF treatment for $1 \mathrm{~h}$, more pulses were applied to the sample with the increase of frequency, thus making it more likely for dislocation proliferation and movement to be initiated, which was verified by the numerous dislocations observed during PMF treatment with a frequency of $10.0 \mathrm{~Hz}$. Furthermore, the temperature rise induced by Joule heat increased as the frequency increased, thus enhancing the irregular motion of atoms and more easily releasing lattice distortion caused by internal stress [17, 37]. Besides, the energy of the atom near the dislocation increased, decreasing the pinning effect on the dislocations and diminishing the resistance of dislocation slip. Therefore, dislocation is easier to move, 

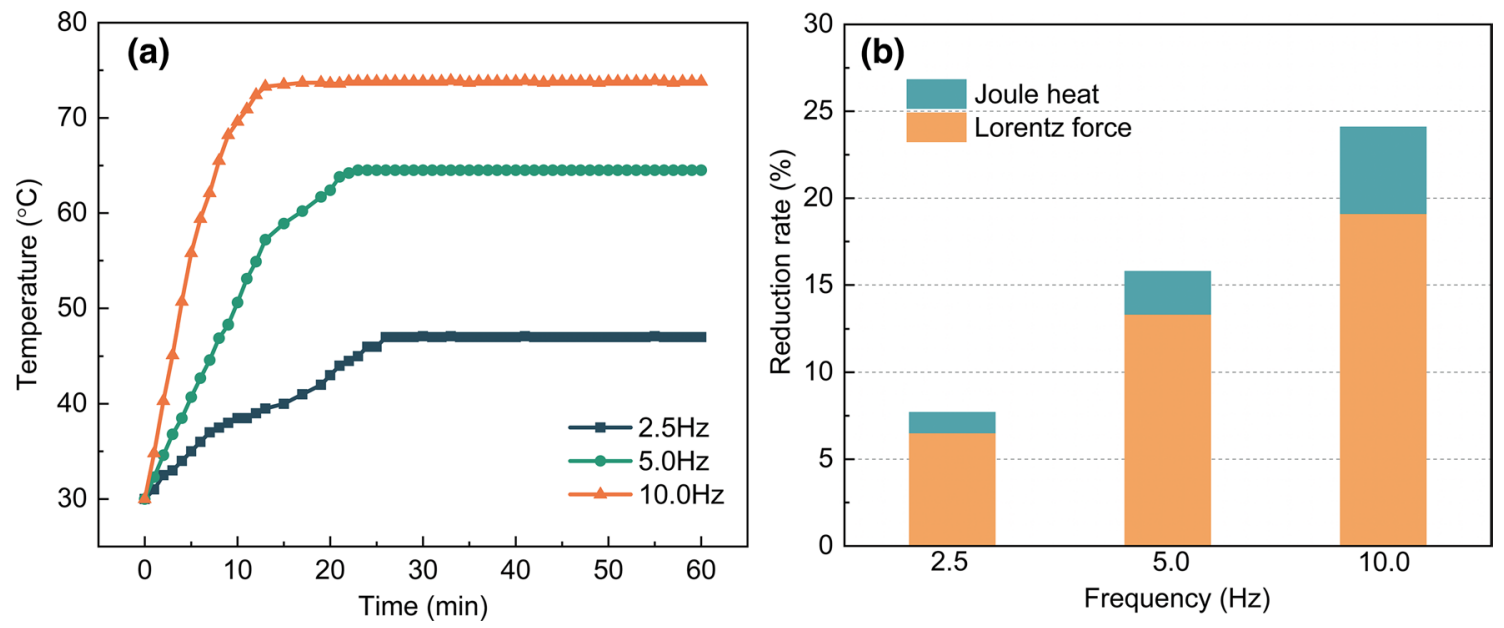

Fig. 10 a Temperature rise during PMF treatment process and $\mathbf{b}$ residual stress elimination caused by Lorentz force and Joule heat

Table 2 Residual stress of samples treated at different temperatures

\begin{tabular}{|c|c|c|c|}
\hline \multirow[t]{2}{*}{ Temperature } & \multicolumn{2}{|c|}{ Residual stress (MPa) } & \multirow{2}{*}{$\begin{array}{l}\text { Reduc- } \\
\text { tion rate } \\
(\%)\end{array}$} \\
\hline & Before treatment & After treatment & \\
\hline $47.0^{\circ} \mathrm{C}$ & $-50.1 \pm 2.2$ & $-49.5 \pm 1.5$ & 1.2 \\
\hline $64.5^{\circ} \mathrm{C}$ & $-49.5 \pm 1.8$ & $-48.2 \pm 1.4$ & 2.6 \\
\hline $73.8^{\circ} \mathrm{C}$ & $-48.7 \pm 2.0$ & $-46.2 \pm 1.2$ & 5.1 \\
\hline
\end{tabular}

and plastic deformation is likely to be generated under the effect of Lorentz force. As a consequence, the residual stress was most remarkably eliminated after PMF treatment with a frequency of $10.0 \mathrm{~Hz}$.

In general, the experimental results of this study show that the residual stress of the rolled magnesium alloy AZ31 sheet can be effectively eliminated by pulsed magnetic field treatment. What is more, the surface quality of the sample will not be damaged during treatment due to the non-contact feature of the PMF device with the sample. Therefore, the pulsed magnetic field may have a promising application prospect in producing sheets while eliminating residual stress.

\section{Conclusions}

This work investigated the effect of pulsed magnetic field on the elimination of the residual stress in the rolled magnesium alloy AZ31 sheet and studied the residual stress and microstructure before and after PMF treatment. The following conclusions can be drawn:

1. The residual stress along the RD and TD of the rolled magnesium alloy AZ31 sheet is effectively eliminated by the pulsed magnetic field. The residual stress is most remarkably eliminated after PMF treatment with a frequency of $10.0 \mathrm{~Hz}$, with an average and maximum reduction rate of $26.6 \%$ and $30.3 \%$, respectively.

2. The Lorentz force facilitates dislocation movement and results in local plastic deformation. Consequently, the strain is homogeneously distributed, thus eliminating the residual stress. The Lorentz force plays a leading role in the process while Joule heat is also conducive to the elimination of residual stress.

Acknowledgement This work was financially supported by the National Key R\&D Program of China (No. 2016YFB0301105).

\section{References}

[1] T. Xu, Y. Yang, X. Peng, J. Song, F.S. Pan, J. Magnes. Alloys 7, 536 (2019)

[2] H. Zengin, Y. Turen, M.E. Turan, F. Aydın, Acta Metall. Sin. (Engl. Lett.) 32, 1309 (2019)

[3] T. Tu, X.H. Chen, J. Chen, C.Y. Zhao, F.S. Pan, Acta Metall. Sin. (Engl. Lett.) 32, 23 (2019)

[4] S.J. Meng, H. Yu, S.D. Fan, Q.Z. Li, S.H. Park, J.S. Suh, Y.M. Kim, X.L. Nan, M.Z. Bian, F.X. Yin, W.M. Zhao, B.S. You, K.S Shin, Acta Metall. Sin. (Engl. Lett.) 32, 145 (2019)

[5] Y. Bai, W.L. Cheng, S.C. Ma, J. Zhang, C. Guo, Y. Zhang, Acta Metall. Sin. (Engl. Lett.) 31, 487 (2018)

[6] W.C. Dong, D.B. Gao, S.P. Lu, Acta Metall. Sin. (Engl. Lett.) 32, 618 (2019)

[7] P.J. Withers, H.K.D.H. Bhadeshia, Mater. Sci. Technol. 17, 366 (2001)

[8] P.J. Withers, Rep. Prog. Phys. 70, 2211 (2007)

[9] S.Q. Xiang, X.F. Zhang, Acta Metall. Sin. (Engl. Lett.) 33, 281 (2020)

[10] T. Hosaka, S. Yoshihara, I. Amanina, B.J. MacDonald, Procedia Eng. 184, 432 (2017) 
[11] B. Chen, A. Skouras, Y.Q. Wang, J.F. Kelleher, S.Y. Zhang, D.J. Smith, P.E.J. Flewitt, M.J. Pavier, Mater. Sci. Eng. A 590, 374 (2014)

[12] J.S. Wang, C.C. Hsieh, C.M. Lin, E.C. Chen, C.W. Kuo, W. Wu, Mater. Sci. Eng. A 605, 98 (2014)

[13] M.C. Sun, Y.H. Sun, R.K. Wang, Mater. Lett. 58, 299 (2004)

[14] X.C. Zhao, Y.D. Zhang, H.W. Zhang, Q. Wu, Acta Metall. Sin. (Engl. Lett.) 21, 289 (2008)

[15] D.A. Lados, D. Apelian, L. Wang, Mater. Sci. Eng. A 527, 3159 (2010)

[16] W.D.S. Mattos, G.E. Totten, L.D.C.F. Canale, Mater. Perform. Charact. 6, 894 (2017)

[17] Y. Lian, P. Ji, J. Zhang, X. Yuan, W. Xu, Y. Zhao, J. Mo, L. Zheng, S. Dou, J. Magnes. Alloys 7, 186 (2019)

[18] C. Wang, T. Luo, J. Zhou, Y. Yang, Mater. Sci. Eng. A 722, 14 (2018)

[19] X.P. Ma, Y.S. Yang, B. Wang, Int. J. Heat Mass Transf. 52, 5285 (2009)

[20] A.L. Lu, F. Tang, X.J. Luo, J.F. Mei, H.Z. Fang, J. Mater. Process. Technol. 74, 259 (1998)

[21] B.E. Klamecki, J. Mater. Process. Technol. 141, 385 (2003)

[22] S. Wu, A. Lu, H. Zhao, H. Fang, F. Tang, Mater. Sci. Eng. A 328, 133 (2002)

[23] Z.P. Cai, X. Huang, Mater. Sci. Eng. A 528, 6287 (2011)
[24] Y.L. Song, L. Hua, J. Mater. Sci. Technol. 28, 803 (2012)

[25] G. Tang, Z. Xu, M. Tang, X. Chen, H. Zhou, A. Lu, Mater. Sci. Eng. A 398, 108 (2005)

[26] X. Yuan, J. Zhang, Y. Lian, C. Du, W. Xu, Y. Zhao, J. Mo, J. Magnes. Alloys 6, 238 (2018)

[27] M. Kamaya, A.J. Wilkinson, J.M. Titchmarsh, Nucl. Eng. Des. 235, 713 (2005)

[28] R.R. Shen, P. Efsing, Ultramicroscopy 184, 156 (2018)

[29] S. Xiang, X. Zhang, Mater. Sci. Eng. A 761, 138026 (2019)

[30] Q. Shao, J. Kang, Z. Xing, H. Wang, Y. Huang, G. Ma, H. Liu, J. Magn. Magn. Mater. 476, 218 (2019)

[31] L.P. Ma, W.X. Zhao, Z.Q. Liang, X.B. Wang, L.J. Xie, L. Jiao., T.F. Zhou, Mater. Sci. Eng. A 609, 16 (2014)

[32] K.L. Zhang, Y.J. Li, Y.S. Yang, Acta Metall. Sin. (Engl. Lett.) (2020). https://doi.org/10.1007/s40195-020-01048-6

[33] K.L. Zhang, Y.J. Li, Y.S. Yang, J. Mater. Sci. Technol. 48, 9 (2020)

[34] W.B. Hutchinson, M.R. Barnett, Scr. Mater. 63, 737 (2010)

[35] H.L. Kim, J.S. Park, Y.W. Chang, Mater. Sci. Eng. A 540, 198 (2012)

[36] J. Wang, J.M. Molina-Aldareguía, J. Llorca, Acta Mater. 188, 215 (2020)

[37] A. Lombardi, D. Sediako, A. Machin, C. Ravindran, R. MacKay, Mater. Sci. Eng. A 697, 238 (2017) 